18

\title{
Спектральный отклик микрорезонатора с двумя квантовыми точками, обусловленный взаимодействием между локализованными электронами
}

\author{
(C) A.В. Цуканов \\ Физико-технологический институт им. К.А. Валиева РАН, \\ 117218 Москва, Россия \\ e-mail: a-v-ts@mail.ru
}

Поступила в редакцию 25.08.2021 г.

В окончательной редакции 15.10.2021 г.

Принята к публикации. 22.10.2021 г.

Рассмотрена теоретическая модель полупроводниковой наноструктуры, состоящей из одномодового микрорезонатора с двумя квантовыми точками. Показано, что кулоновское взаимодействие между электронами, локализованными в квантовых точках, модифицирует спектральный отклик системы на внешнее лазерное поле. Обсуждена возможность ее использования для детектирования элементарного заряда в третьей (оптически неактивной) квантовой точке. Изучено влияние как диагональных (эффект Штарка), так и недиагональных (эффект Ферстера) кулоновских матричных элементов гамильтониана на точность детектирования. Рассчитаны зависимости измерительного контраста от параметров резонатора и квантовых точек. Установлено существование таких структурных конфигураций, для которых контраст сохраняет оптимальное значение даже при больших расстояниях до измеряемой точки.

Ключевые слова: квантовые точки, микрорезонаторы, нанофотоника, полупроводники, лазер, измерение.

DOI: $10.21883 / \mathrm{OS} .2022 .02 .52002 .2678-21$

\section{Введение}

Наноструктуры, состоящие из полупроводниковых квантовых точек (КТ) [1], часто рассматриваются в качестве элементарных носителей информации нового поколения. Массивы одноэлектронных КТ предлагается использовать для хранения, перемещения и обработки данных как в классических, так и в квантовых вычислительных устройствах [2-4]. Кроме того, существуют схемы высокотехнологичных приборов, также имеющих отношение к практическому применению КТ в современных информационных и коммуникационных сетях [5]. В их число входят источники одиночных фотонов [6] и коррелированных фотонных пар [7], клеточные автоматы [8], преобразователи фазы и частоты $[9,10]$, а также сенсорные устройства - одноэлектронные транзисторы $[11,12]$ и квантовые точечные контакты [13]. Принцип действия емкостных сенсоров основан на зависимости положения энергетических уровней электрона в КТ, входящей в прибор, а значит, и квантового наноамперного тока через КТ от внешнего электрического поля.

Кулоновское взаимодействие электронов КТ между собой и с внешними заряженными системами модифицирует состояние прибора, а значит, может служить в качестве дополнительного ресурса для оптимизации процедуры измерения. Основная особенность электронэлектронного взаимодействия (в отличие, скажем, от электрон-фотонных эффектов) состоит в том, что его нельзя выключить. Это необходимо учитывать при создании квантовых регистров, поскольку кулоновский сдвиг электронных уровней данного КТ-кубита в поле электронов соседних кубитов приводит к дополнительному фазовому сдвигу его состояния, что требует применения дополнительных корректирующих алгоритмов. Не менее тщательно следует подходить к проектированию сенсоров, где используются заряженные КТ.

В настоящей работе рассматривается модель гибридной оптоэлектронной системы, где в отличие от традиционной схемы измерения заряда анализируется зависимость не электрического тока, протекающего от источника к стоку непосредственно через КТ, а субфотонного сигнала, проходящего от лазера к фотоприемнику через микрорезонатор (МР) [14]. Поскольку фотоны не взаимодействуют с заряженным объектом вне МР, то необходимо организовать эффективный контакт между ними, дополнив схему элементом-трансдюсером. В качестве такого элемента можно взять одну или несколько заряженных КТ, расположенных в пучностях моды МР для обмена с ней квантом энергии и при этом взаимодействующих с объектом согласно закону Кулона. Таким образом, реализуется непрямая связь между фотонами, идущими от источника к детектору через МР, и заряженным объектом [15]. Здесь в роли объекта выступает КТ, находящаяся на значительном удалении от МР. В ходе теоретического исследования было получено аналитическое выражение для отклика системы с двумя КТ-трансдюсерами в субфотонном режиме. Исследовано влияние штарковской и ферстеровской компонент на измерительный контраст в рамках формализма матрицы плотности. Показано, что усиление энергетического об- 
мена Ферстера между резонансными КТ при их сближении может способствовать поддержанию высоких значений измерительного контраста даже для удаленных КТ.

\section{Модель оптоэлектронной структуры и основные уравнения}

Необходимым компонентом многих квантово-оптических устройств являются фотонные кристаллы (ФК), представляющие собой стержни или пластины с литографически изготовленными вертикальными отверстиями [16]. Эти отверстия формируют упорядоченную брэгговскую структуру (решетку), позволяющую локализовать фотонное поле внутри ФК. Спектр ФК разбивается на запрещенные и разрешенные зоны (частотные интервалы с квазинепрерывным распределением собственных мод). При этом дефекты решетки - пропущенные отверстия - помогают осуществить дальнейшую инженерию спектральных свойств ФК. Протяженные одномерные дефекты могут функционировать в качестве волноводов, связывающие КТ-структуру с источником или детектором фотонов. Дефекты, образованные всего несколькими пропущенными отверстиями, представляют собой микрорезонаторы с дискретным набором собственных мод. Структуры на основе ФК применяются как оптические сенсоры давления, температуры, механических напряжений и химического состава исследуемых образцов [17]. Однако для их использования в роли детекторов электрических полей и отдельных элементарных зарядов необходимо организовать взаимодействие фотонов, транспортируемых через ФК, с данными полями (зарядами). Сами по себе они не взаимодействуют, поэтому для этой цели используется специальный элемент схемы - трансдюсер, который одновременно связан с обеими подсистемами.

Мы рассмотрим одномерный ФК с дефектом решетки (MP), который поддерживает изолированные моды с частотами, близкими к частотам электронных переходов в полупроводниковых КТ (рис. 1). Если в области высокодобротного МР находится одна КТ, частота которой соответствует частоте моды МР, то система описывается хорошо известной моделью Джейнса-Каммингса, демонстрируя осцилляции Раби (периодическая трансформация электронного возбуждения КТ в фотон МР и обратно). Если же МР содержит две и более таких КТ, то эволюция данной системы будет определяться соотношением характерных энергий электрон-фотонного и кулоновского взаимодействий. Именно эти КТ, частоты которых, с одной стороны, зависят от внешних полей и зарядов, а с другой стороны, влияют на прохождение фотонов через МР, могут выполнять функции трансдюсера.

Пусть внутри МР находятся две КТ, каждая из которых содержит по два одноэлектронных состояния (основное и возбужденное). Основное состояние $\left|g_{k}\right\rangle$ КТ с номером $k(k=1,2)$ имеет энергию $\varepsilon_{g, k}$, a ее возбужденное состояние $\left|e_{k}\right\rangle-$ энергию $\varepsilon_{e, k}$. Лазер с частотой $\omega_{L}$ фокусируется на входном порте ФК. Скорость $\Omega_{L}$ поступления фотонов в структуру определяется степенью перекрытия полей моды ФК и лазера. Будем считать, что частота $\omega_{c}$ моды МР и частоты КТ 1 и КТ 2 достаточно близки, чтобы можно было говорить о возможности резонансного фотонного обмена между ними. Третья КТ, электронная заселенность которой подлежит определению, имеет одно (основное) состояние $\left|g_{3}\right\rangle$ с энергией $\varepsilon_{g, 3}$. Предполагается, что мощность потенциального барьера, разделяющего КТ, велика настолько, что туннельными и обменными эффектами можно пренебречь: каждый электрон остается локализованным в своей КТ.

Если принять за начало отсчета энергию $\varepsilon_{g, 1}$, то гамильтониан МР и трех одноэлектронных КТ, которые не взаимодействуют между собой, имеет вид (здесь и далее полагаем $\hbar \equiv 1)$ :

$$
\begin{aligned}
H_{0}= & \omega_{c} a^{\dagger} a+\Delta_{2,1}\left|g_{2}\right\rangle\left\langle g_{2}\left|+\Delta_{3,1}\right| g_{3}\right\rangle\left\langle g_{3}\left|+\omega_{1}\right| e_{1}\right\rangle\left\langle e_{1}\right| \\
& +\left(\omega_{2}+\Delta_{2,1}\right)\left|e_{2}\right\rangle\left\langle e_{2}\right|,
\end{aligned}
$$

где $\omega_{k}=\varepsilon_{e, k}-\varepsilon_{g, k}-$ частоты электронных переходов КТ, $\Delta_{2,1}=\varepsilon_{g, 2}-\varepsilon_{g, 1}$ и $\Delta_{3,1}=\varepsilon_{g, 3}-\varepsilon_{g, 1}-$ разности энергий основных состояний КТ. Штарковские сдвиги уровней КТ, обусловленные кулоновским взаимодействием электронов и равные диагональным матричным элементам соответствующего оператора, учитываются с помощью гамильтониана

$$
\begin{aligned}
& H_{S}=V_{1,2}^{g g}\left|g_{1} g_{2}\right\rangle\left\langle g_{1} g_{2}\left|+V_{1,3}^{g g}\right| g_{1} g_{3}\right\rangle\left\langle g_{1} g_{3}\right| \\
& +V_{2,3}^{g g}\left|g_{2} g_{3}\right\rangle\left\langle g_{2} g_{3}\left|+V_{1,2}^{e g}\right| e_{1} g_{2}\right\rangle\left\langle e_{1} g_{2}\left|+V_{1,3}^{e g}\right| e_{1} g_{3}\right\rangle\left\langle e_{1} g_{3}\right| \\
& +V_{2,1}^{e g}\left|g_{1} e_{2}\right\rangle\left\langle g_{1} e_{2}\left|+V_{2,3}^{e g}\right| e_{2} g_{3}\right\rangle\left\langle e_{2} g_{3}\right| .
\end{aligned}
$$

Недиагональные кулоновские матричные элементы описывают резонансный обмен квантом возбуждения между КТ 1 и КТ 2 без перемещения электрона (эффект Ферстера [18]):

$$
H_{F}=V_{1,2}^{F}\left(\left|g_{1} e_{2}\right\rangle\left\langle g_{2} e_{1}|+| g_{2} e_{1}\right\rangle\left\langle g_{1} e_{2}\right|\right) .
$$

Данный процесс будет эффективным лишь в случае близости частот переходов в обеих КТ, т. е. когда выполняется условие их резонанса $\left|\omega_{1}-\omega_{2}\right| \ll\left|V_{1,2}^{F}\right|$. Матричные элементы в выражениях (2) и (3)

$$
V_{k, m}^{g(e) g}=2 \int d \mathbf{r}_{k} \int d \mathbf{r}_{m}\left|\psi_{g(e), k}\left(\mathbf{r}_{k}\right)\right|^{2}\left|\psi_{g, m}\left(\mathbf{r}_{m}\right)\right|^{2} /\left|\mathbf{r}_{k}-\mathbf{r}_{m}\right|
$$

и

$$
V_{1,2}^{F}=2 \int d \mathbf{r}_{1} \int d \mathbf{r}_{2} \psi_{e, 1}^{*}\left(\mathbf{r}_{1}\right) \psi_{g, 2}^{*}\left(\mathbf{r}_{2}\right) \psi_{g, 1}\left(\mathbf{r}_{1}\right) \psi_{e, 2}\left(\mathbf{r}_{2}\right) /\left|\mathbf{r}_{1}-\mathbf{r}_{2}\right|
$$

(далее $V_{1,2}^{F} \equiv V_{F}$ ) представляют собой пространственные интегралы, зависящие от вида одноэлектронных волновых функций, от расстояния между центрами КТ и от взаимной ориентации КТ. В качестве единиц измерения здесь приняты эффективные атомные 


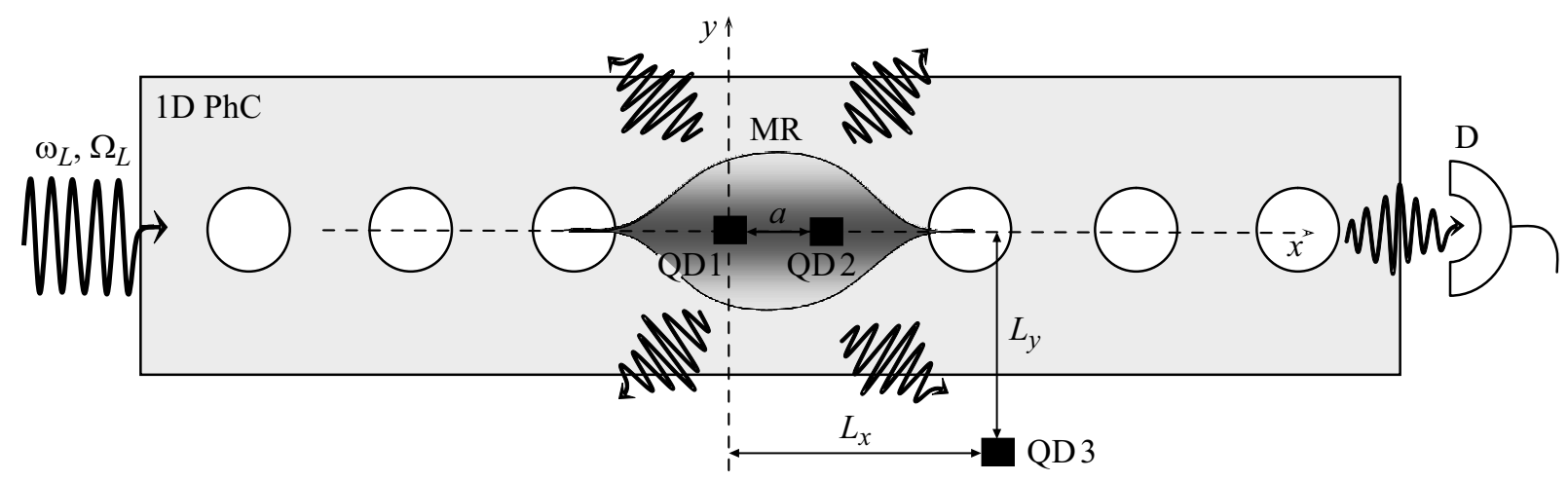

Рис. 1. Схема одномерного фотонного кристалла с дефектом (микрорезонатором), содержащим две квантовые точки. Их центры расположены на оси симметрии $x$ в пучностях поля моды МР на расстоянии $a$ друг от друга. Начало отсчета координат совмещено с центром первой КТ. Лазер осуществляет накачку системы фотонами, которые, проходя через МР, взаимодействуют с двумя КТ, а затем попадают в детектор или рассеиваются. Третья (измеряемая) КТ с координатами центра $\left(L_{x}, L_{y}\right)$, расположенная вне МР, не взаимодействует с фотонами напрямую, однако влияет на частоту перехода в других (сенсорных) КТ за счет кулоновского взаимодействия между ними.

единицы: 1 e.a.u. $=R y^{*}=m^{*} R y / m_{e} \varepsilon^{2}$ для энергии и 1 e.a.u. $=a_{B}^{*}=a_{B} m_{e} \varepsilon / m^{*}$ для длины, где $R y=13.6 \mathrm{eV}-$ энергия Ридберга, $a_{B}=0.52 \cdot 10^{-10} \mathrm{~m}$ - радиус Бора, $m_{e}$ - масса свободного электрона, $m^{*}-$ эффективная масса электрона, $\varepsilon$ - электрическая проницаемость полупроводника. Для арсенида галлия (GaAs: $\varepsilon=12$ и $\left.m^{*}=0.067 m_{e}\right)$ имеем $R y=6 \mathrm{meV}$ и $a_{B}^{*}=10 \mathrm{~nm}$. Процессы когерентного электрон-фотонного обмена Джейнса-Каммингса между КТ и модой МР, а также накачка моды МР фотонами лазера описываются гамильтонианами

$$
\begin{aligned}
H_{J C}= & -\Omega_{1}\left(\left|e_{1}\right\rangle\left\langle g_{1}|a+| g_{1}\right\rangle\left\langle e_{1}\right| a^{\dagger}\right) \\
& -\Omega_{2}\left(\left|e_{2}\right\rangle\left\langle g_{2}|a+| g_{2}\right\rangle\left\langle e_{2}\right| a^{\dagger}\right)
\end{aligned}
$$

и

$$
H_{L}=2 \Omega_{L} \cos \left(\omega_{L} t\right)\left(a^{\dagger}+a\right) .
$$

При выводе уравнения (4) использовано приближение вращающейся волны, предполагающее выполнение условий $\omega_{k} \gg \Omega_{k}$. Таким образом, гамильтониан нашей системы представляется в виде суммы гамильтонианов (1)-(5):

$$
H=H_{0}+H_{S}+H_{F}+H_{J C}+H_{L} .
$$

Кроме того, необходимо учесть некогерентные процессы фотонной диссипации, связанные с неконтролируемым уходом энергии из МР в континуум со скоростью $\kappa$, и электронную релаксацию со скоростями $\gamma_{k}$, обусловленную взаимодействием $k$-й КТ с фононным окружением. Строгий учет этих явлений возможен лишь в рамках формализма матрицы плотности и уравнения Линдблада (см. ниже), однако приближенное решение, справедливое для небольшой $(\leq 0.1)$ вероятности возбуждения системы из вакуумного состояния, может быть найдено с помощью более простого (в вычислительном отношении) формализма уравнения Шредингера.
Для этого в выражении (1) следует произвести замену $\omega_{c} \rightarrow \omega_{c} \rightarrow i \kappa$ и $\omega_{k} \rightarrow \omega_{k}-i \gamma_{k}$.

Если предположить, что мощность лазерной накачки невелика $\left(\Omega_{L} \leq \kappa\right)$, то можно считать, что в структуре присутствует не более одного кванта возбуждения. Тогда пространство состояний сводится к четырем базисным векторам. Вектор $|1\rangle=\left|g_{1}, g_{2}, g_{3}\right\rangle\left|0_{c}\right\rangle$ отвечает вакуумному состоянию электрон-фотонной системы, векторы $|2\rangle=\left|e_{1}, g_{2}, g_{3}\right\rangle\left|0_{c}\right\rangle$ и $|3\rangle=\left|g_{1}, e_{2}, g_{3}\right\rangle\left|0_{c}\right\rangle$ описывают возбуждение электрона в КТ 1 и КТ 2, а вектор $|4\rangle=\left|g_{1}, g_{2}, g_{3}\right\rangle\left|1_{c}\right\rangle$ - нахождение одного фотона в моде МР. Вектор состояния $|\Psi\rangle=\sum_{k=1}^{4} c_{k}|k\rangle$ системы представляется в виде разложения по базисным векторам с коэффициентами $c_{k}$, зависящими от времени. Эволюция вектора состояния подчиняется уравнению Шредингера $i \partial_{t}|\Psi\rangle=H|\Psi\rangle$ с начальным условием $|\Psi(0)\rangle=|1\rangle$. Интересующее нас установившееся решение (средняя фотонная заселенность МР) в системе отсчета, связанной с лазером, имеет вид

$$
\begin{gathered}
\langle n\rangle=\left|c_{4}\right|^{2} \approx \frac{\Omega_{L}^{2}\left|\Delta_{F}\right|^{2}}{\mid\left(\delta_{c}-i \kappa\right) \Delta_{F}-\Omega_{1}^{2}\left(\delta_{2}-\Delta_{2,1}+G_{2}-i \gamma_{2}\right)}, \\
-\Omega_{2}^{2}\left(\delta_{1}+G_{1}-i \gamma_{1}\right)+\left.2 V_{F} \Omega_{1} \Omega_{2}\right|^{2} \\
\Delta_{F}=\left(\delta_{1}+G_{1}-i \gamma_{1}\right)\left(\delta_{2}-\Delta_{2,1}+G_{2}-i \gamma_{2}\right)-V_{F}^{2},
\end{gathered}
$$

где введены отстройки частот МР и КТ от лазерной частоты: $\delta_{c}=\omega_{c}-\omega_{L}$ и $\delta_{k}=\omega_{k}-\omega_{L}$, а также определены штарковские сдвиги частот КТ 1 и КТ 2: $G_{1}=V_{1,2}^{e g}-V_{1,2}^{g g}+V_{1,3}^{e g}-V_{1,3}^{g g}$ и $G_{2}=V_{2,1}^{e g}-V_{2,1}^{g g}+V_{2,3}^{e g}-V_{2,3}^{g g}$ соответственно. При отсутствии взаимодействия между КТ и МР, когда $\left|\Omega_{k} / \delta_{k}\right| \ll 1$, отклик системы совпадает с лоренцевой кривой для „пустого“ МР. Если оптически активна только КТ $1\left(\left|\Omega_{1} / \delta_{1}\right| \gg 1\right.$, $\left.\left|\Omega_{2} / \delta_{2}\right| \ll 1\right)$, а кулоновскими эффектами можно пренебречь $\left(\left|V_{F} / \Omega_{1}\right|,\left|G_{1} / \Omega_{1}\right| \ll 1\right)$, то кривая отклика имеет два поляритонных пика, предсказываемые моделью 
Джейнса-Каммингса. Подключение КТ 2 в резонанс с модой МР и КТ $1\left(\left|\Omega_{2} / \delta_{2}\right| \gg 1,\left|G_{2} / \Omega_{2}\right| \ll 1\right)$ в данных условиях обеспечивает увеличение расщепления поляритонного дублета в $\sqrt{2}$ раз в соответствии с моделью Тависа-Каммингса [15]. Пространственное сближение двух КТ приводит к усилению влияния кулоновских эффектов на спектр и динамику. Когда сдвиги $G_{1}$ и $G_{2}$ оказываются сопоставимыми с частотами Раби, начинается переход системы в дисперсионный режим. При этом восстанавливается кривая отклика „Пустого“ МР, а влияние КТ сохраняется в дисперсионном сдвиге $\sim\left(G_{1}^{2} / \Omega_{1}+G_{2}^{2} / \Omega_{2}\right)$ частоты пика. Наконец, учет ферстеровской компоненты в условиях резонанса частот переходов КТ снимает их вырождение, генерируя расщепление порядка $2 V_{F}$. В случае, когда энергии ферстеровского и оптического взаимодействий примерно одинаковы и превосходят отстройки и сдвиги частот, имеет место гибридизация фотона МР и коррелированных электронных возбуждений в группе КТ. Необходимо помнить, что штарковские и ферстеровские вклады зависят от расстояния между центрами КТ и являются, таким образом, взаимосвязанными. Однако сдвиги частот КТ, обусловленные взаимодействием их электронов, могут быть скомпенсированы за счет привлечения дополнительного (независимого) ресурса внешнего электростатического поля, создаваемого затворами вблизи КТ. Ниже мы исследуем влияние на фотонный спектр кулоновских вкладов, фокусируя внимание на эффекте Ферстера, а также вычислим измерительный контраст, варьируя расстояние от структуры до измеряемой КТ.

\section{Влияние эффекта Ферстера на спектральный отклик системы}

Для изучения спектрального отклика структуры необходимо рассчитать энергии $G_{1}, G_{2}$ и $V_{F}$. Воспользуемся экспоненциально-степенным приближением для потенциала двумерной КТ [19]:

$$
U(x, y)=U_{0} \exp \left[-\left(x / r_{x}\right)^{2 p}-\left(y / r_{y}\right)^{2 p}\right],
$$

где $U_{0}$ - глубина потенциала КТ, $2 r_{x(y)}$ - длина КТ вдоль направления $x(y)$, а $p$ - параметр, задающий гладкость потенциала. При $p \geq 3$ форма такой КТ близка к прямоугольной. Выберем параметры потенциала КТ (8) следующим образом: $r_{x}=0.7, r_{y}=0.8$, $U_{0}=-22$ и $p=5$. Частота перехода $\omega_{Q D}=\omega_{1(2)}$ между основным и возбужденным состояниями КТ, соответствующая частоте МР в условиях резонанса, составляет примерно 10 e.a.u., и в дальнейшем мы будем пользоваться данной частотой в качестве единицы измерения энергии. Найдя волновые функции $\psi_{g, k}(\mathbf{r})$ и $\psi_{e, k}(\mathbf{r})$ одноэлектронных состояний, построим зависимости матричных элементов $G_{1}, G_{2}$ и $V_{F}$ от расстояния $a$ между центрами двух одинаковых КТ и угла поворота $\varphi_{2}$ КТ 2 относительно ее центра в отсутствие внешней КТ 3 (рис. 2). Сдвиг частоты КТ 1 $G_{1}$ практически не меняется при повороте КТ 2, находящейся в основном $s$-состоянии, волновая функция которого характеризуется слабой угловой зависимостью. Сдвиг частоты вращаемой КТ 2 хорошо описывается формулой $G_{2} \approx \frac{a_{p}^{2}}{a^{3}}\left(2-3 \sin ^{2} \varphi_{2}\right)$, полученной в классическом приближении точечных зарядов для эффективного радиуса возбужденного $p$-состояния $a_{p}=0.56$. Зависимость энергии Ферстера имеет особенность, которая не может быть объяснена с помощью классических представлений, как в случае со штарковскими сдвигами. На графике видно, что при повороте КТ 2 на угол $\pi$ и восстановлении исходной плотности распределения заряда двух КТ энергия Ферстера принимает значение, равное по амплитуде, но противоположное по знаку своему начальному значению, $V_{F}(\pi)=-V_{F}(0)$. Чтобы получить исходное значение данного параметра, необходимо совершить полный поворот на угол $2 \pi$. С ростом расстояния $a$ между центрами КТ наблюдается быстрый спад $\left(\sim 1 / a^{3}\right)$ всех зависимостей.

Формула (7), полученная с помощью уравнения Шредингера, может быть использована для качественного анализа отклика структур, в которых дефазировка электронного состояния КТ не является преобладающим каналом потери когерентности. В общем случае необходимо применить уравнение Линдблада, решение которого дает зависимость матрицы плотности $\rho(t)$ от времени, для заданного начального состояния $\rho(0)$ :

$$
\begin{aligned}
\frac{d \rho}{d t}= & -i[H, \rho]+\kappa D(a)+\sum_{k=1}^{2} \gamma_{k} D\left(\left|g_{k}\right\rangle\left\langle e_{k}\right|\right) \\
& +\sum_{k=1}^{2} \gamma_{d, k} D\left(\left|e_{k}\right\rangle\left\langle e_{k}\right\rangle-\left|g_{k}\right\rangle\left\langle g_{k}\right|\right),
\end{aligned}
$$

где $\gamma_{d, k}-$ скорость дефазировки электрона в $k$ й КТ. Диссипативные фотонные и электронные процессы моделируются супероператорами Линдблада $D(O)=O \rho O^{\dagger}-\left[O^{\dagger} O, \rho\right] / 2$. Как показывает сравнение графиков зависимостей среднего числа фотонов в МР от частоты лазера, полученных с помощью приближения (7) и уравнения (9), резонансные частоты совпадают для обоих типов данных. Учет дефазировки приводит к дополнительному уширению и уменьшению амплитуды пиков. Таким образом, аналитическая формула (7) дает корректные значения собственных частот системы как функций энергий Штарка и Ферстера.

Мы будем рассматривать компактную структуру с небольшим расстоянием между КТ, а значит, обладающую заметной энергией ферстеровского обмена, $V_{F} \geq \Omega_{1,2}$. Кроме того, мы будем предполагать, что штарковские внутриструктурные сдвиги компенсируются за счет выбора глубин потенциалов КТ. В этом случае спектр двух одноэлектронных КТ при условии близости их частот представляет собой дублет с 

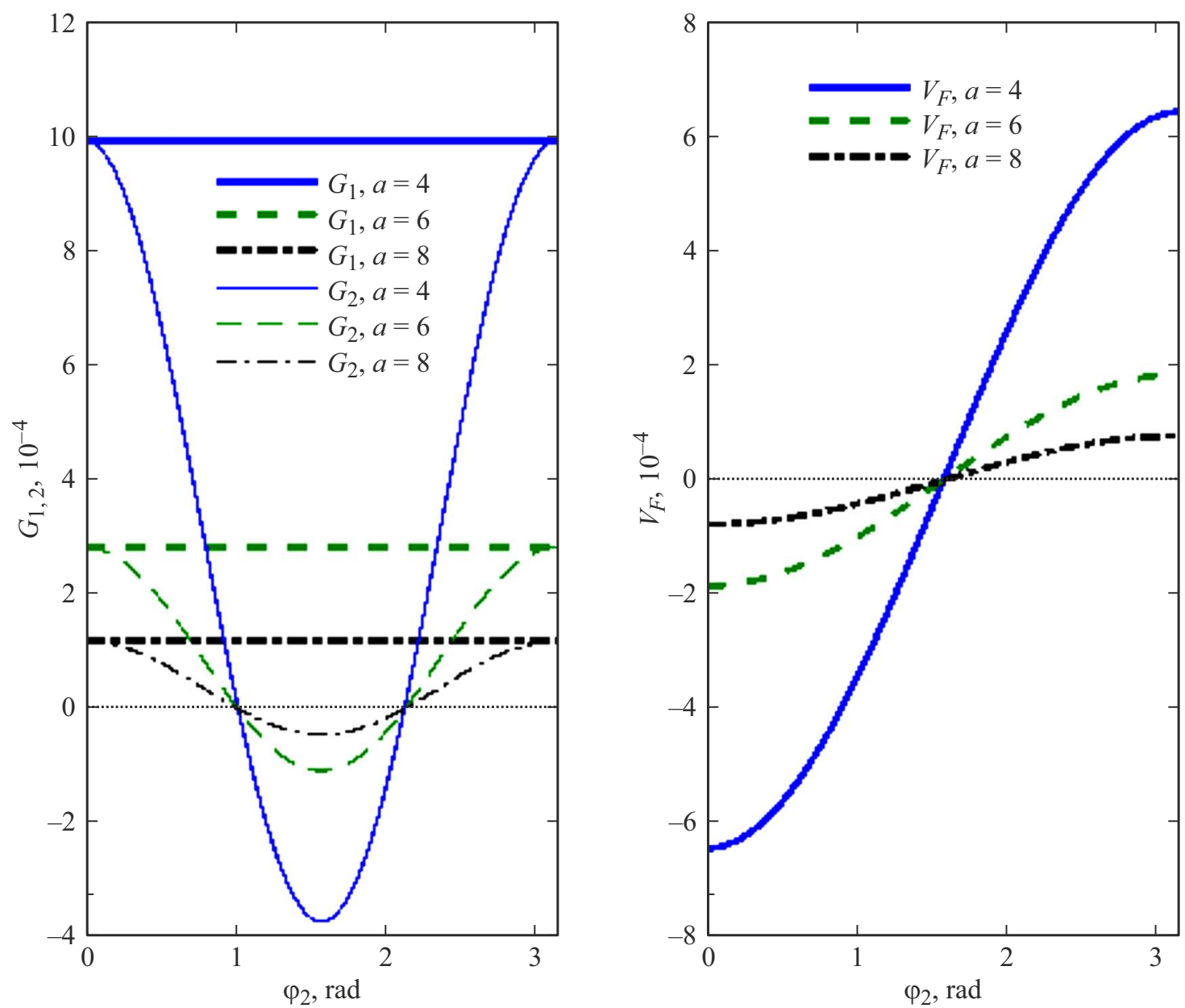

Рис. 2. Графики зависимостей энергий Штарка (слева) и Ферстера (справа) от угла поворота $\varphi_{2}$ КТ 2 вокруг оси $z$, проходящей через ее центр, для трех значений расстояния между центрами КТ 1 и КТ 2. Энергии даны в единицах частоты перехода в КТ 1(2), а расстояния - в эффективных атомных единицах.
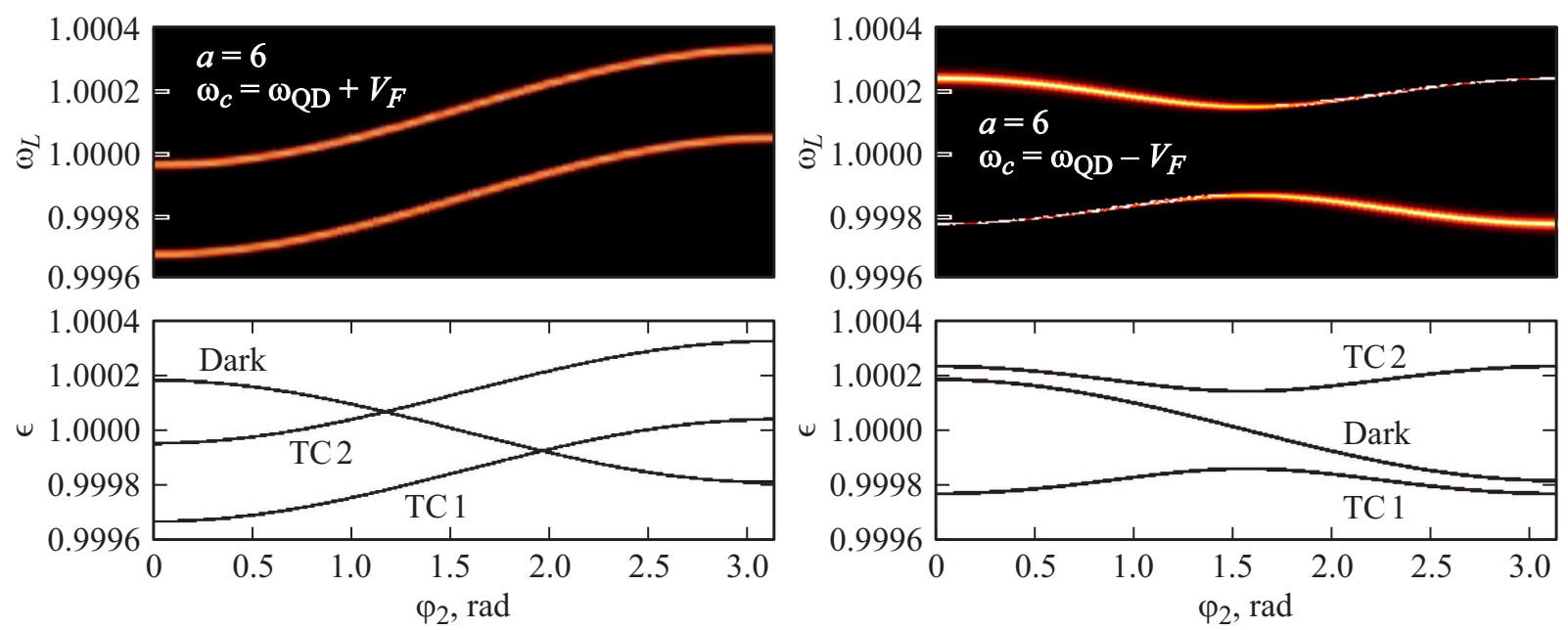

Рис. 3. Графики зависимостей среднего числа фотонов МР (верхняя панель) и энергий электрон-фотонных состояний Тависа-Каммингса и „темного“ состояния (нижняя панель) от угла поворота $\varphi_{2}$ КТ 2 вокруг оси $z$, проходящей через ее центр. Частота МР соответствует частоте одного из дублетов Ферстера. Все величины даны в единицах частоты перехода в КТ 1(2). 
энергиями порядка $\omega_{Q D} \pm V_{F}$. Настройка частоты МР в резонанс с каждой из них приводит к дальнейшему расщеплению и формированию дублетов ТС1 и ТС2 Тависа-Каммингса. Зависимости спектрального отклика системы от угла $\varphi_{2}$, а также собственные энергии гамильтониана (1) без учета мнимых добавок и лазерного поля представлены на рис. 3 для двух резонансов $\omega_{c}=\omega_{Q D} \pm V_{F}$. Можно видеть, что кроме оптически активных состояний с электронной компонентой $(|2\rangle+|3\rangle) / \sqrt{2}$ в спектре присутствует и „темное“ состояние $(|2\rangle-|3\rangle) / \sqrt{2}$, не возбуждаемое внешним полем. Увеличение расстояния между центрами КТ приводит к сглаживанию угловой зависимости и восстановлению спектра Тависа-Каммингса. В отсутствие компенсации сдвигов симметрия гамильтониана понижается, и каждое из собственных состояний содержит светлую компоненту. Здесь и далее в расчетах используется следующий набор параметров: $\Omega_{L}=8 \cdot 10^{-6}, \Omega_{1(2)}=10^{-4}, \kappa=10^{-5}$, $\gamma_{1(2)}=10^{-6}, \gamma_{d, 1(2)}=\gamma_{1(2)} / 2$ и $\Delta_{2,1}=\Delta_{3,1}=0$.

\section{Влияние эффекта Ферстера на измерительный контраст}

Детектирование внешнего заряда с помощью электрон-фотонной структуры, состоящей из одной КТ в МР, основано на разнице значений ее спектрального отклика в отсутствие и при наличии заряда. Внешний заряд вызывает дополнительное штарковское смещение частоты перехода КТ и, как следствие, изменение эффективной энергии взаимодействия КТ и МР. Сравнивая отклик системы $\langle n\rangle$ с калибровочной зависимостью $\left\langle n_{0}\right\rangle$, полученной при отсутствии электрона в измеряемой КТ, можно определить, содержит ли данная КТ электрон или нет. Измерение лучше всего проводить на частоте лазера, для которой разность откликов максимальна. Чтобы количественно охарактеризовать чувствительность структуры к внешним зарядам и полям, мы определим измерительный контраст

$$
S=\max \left|\left(\left\langle n\left(\omega_{L}\right)\right\rangle-\left\langle n_{0}\left(\omega_{L}\right)\right\rangle\right) /\left\langle n_{0}\left(\omega_{L}\right)\right\rangle\right| .
$$

Величина $S$ зависит как от свойств массива КТ и МР, так и от расстояния до тестируемого объекта (в нашем случае - до КТ 3).

Добавление новых резонансных КТ в МР приводит к увеличению расщепления поляритонных пиков дублета Тависа-Каммингса, что должно повышать точность измерения. При этом, однако, частоты КТ смещаются из-за взаимодействия электронов КТ, что может существенно влиять на отклик структуры и в отсутствие внешнего заряда. Кроме того, эффект Ферстера обусловливает гибридизацию электронных состояний резонансных КТ за счет обмена энергией между собой, что также приводит к частотным сдвигам. Как было показано выше, эти внутриструктурные сдвиги могут быть частично

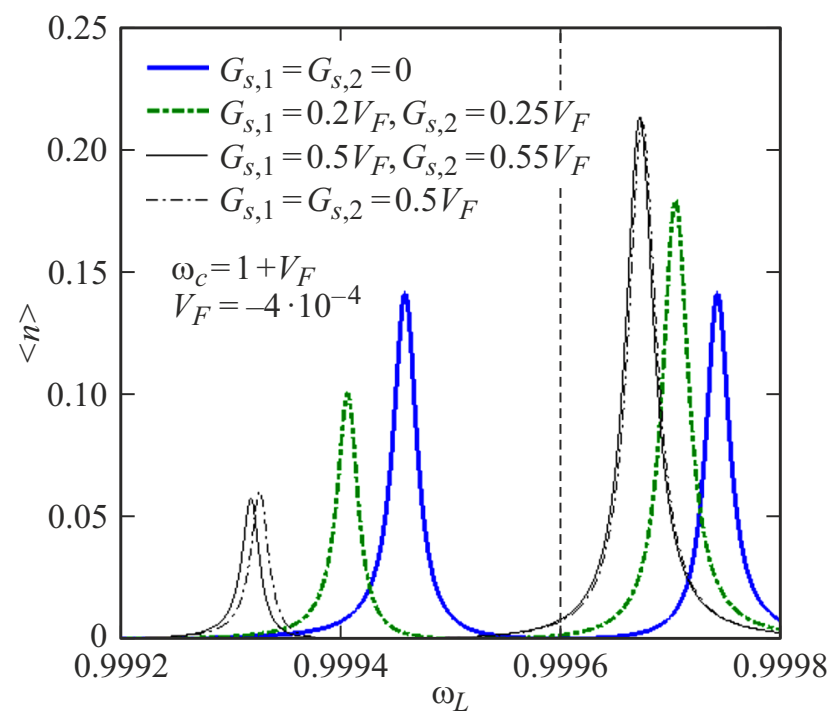

Рис. 4. Спектральный отклик двух одинаковых одноэлектронных КТ в МР в установившемся субфотонном режиме накачки с компенсацией внутриструктурных штарковских сдвигов. Частота MP настроена в резонанс с частотой нижнего гибридизированного двухэлектронного состояния. Положение внешней КТ 3 с электроном задает величины сдвигов частот КТ 1 и КТ 2, от которых зависит форма отклика. Вертикальная штриховая линия отмечает положение частоты МР, не взаимодействующего со структурой. Все величины даны в единицах частоты перехода в КТ 1(2).

скомпенсированы путем выбора параметров КТ (размеров, глубины, ориентации). Если минимизация штарковских сдвигов, ослабляющих связь между электронной и фотонной подсистемами, выглядит обоснованной, то влияние эффекта Ферстера на точность измерения требует более тщательного рассмотрения. На рис. 4 приведены графики спектрального отклика структуры с компенсацией внутренних штарковских сдвигов КТ 1 и КТ 2 в зависимости от внешних штарковских сдвигов $G_{s, k}=V_{k, 3}^{e g}-V_{k, 3}^{g g}$, создаваемых электроном в КТ 3. Энергия Ферстера $V_{F}=-4 \cdot 10^{-4}$ фиксирована, а частота МР соответствует частоте нижнего гибридизированного состояния КТ 1 и КТ 2. В отсутствие электрона в измеряемой КТ 3 отклик представлен двумя симметричными пиками Тависа-Каммингса, смещенными влево и вправо от частоты MP на величину $\Omega_{1(2)} \sqrt{2}$. Взаимодействие электронов, генерирующее малые по сравнению с $\left|V_{F}\right|$ штарковские сдвиги, вызывает амплитудную асимметрию пиков и смещение их частот. С увеличением сдвигов, когда $G_{s, 1(2)} \geq\left|V_{F}\right|$, происходит „диссоциация“ оптической и электронной подсистем из-за нарушения условий резонанса их частот. При этом правый пик трансформируется в пик МР без КТ, а амплитуда левого пика стремится к нулю. В данных условиях в соответствие с определением (10) измерительный контраст принимает значения, близкие к единице. Небольшая разница штарковских сдвигов частот КТ 1 и КТ 2 

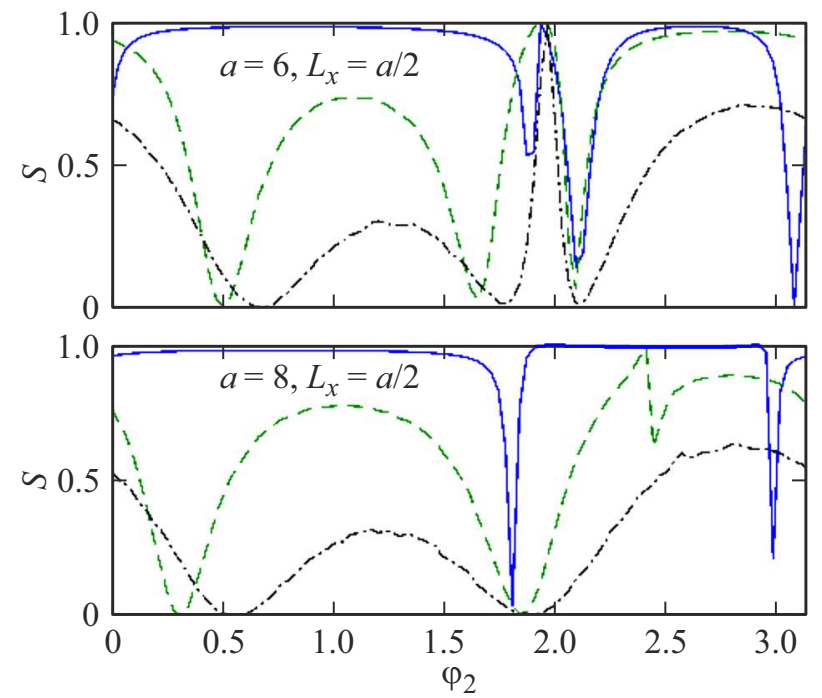

Рис. 5. Графики зависимостей контраста от угла поворота КТ 2 (энергии Ферстера) для расстояния между КТ 1 и КТ 2 $a=6$ (вверху) и $a=8$ (внизу). Измеряемая КТ 3 имеет координаты центра $L_{x}=a / 2$ и $L_{y}=4$ (сплошная линия), $L_{y}=8$ (штриховая линия), $L_{y}=12$ (штрихпунктирная линия).

(тонкие линии на рис. 4) приводит тем не менее к определенной разнице в откликах, что позволяет говорить о возможности использования структуры для дифференциальной электрометрии (отслеживании малых смещений внешнего заряда). Как показывают расчеты, наиболее высокие значения разности откликов получаются при воздействии лазера с частотой, соответствующей резонансной частоте одного из пиков структуры в отсутствие электрона в КТ 3.

Практическую важность имеет вопрос о расстоянии измеряемой КТ 3 до структуры, при котором контраст $S$ сохраняет высокие значения. Пусть данная КТ имеет горизонтальную координату $L_{x}=a / 2$ и смещена по вертикали от оси $x$, соединяющей центры КТ 1 и КТ 2 , на величину $L_{y}$. Положим частоту МР равной частоте $\omega_{Q D}+V_{F}$ двухэлектронного состояния КТ 1 и КТ 2. Частота лазера сканируется в окрестности нижнего состояния ТС1 дублета Тависа-Каммингса (рис. 3) На небольшом удалении КТ $3\left(L_{y} \leq a\right)$ сдвиги $G_{s, 1(2)}$ сопоставимы с величинами $\Omega_{1(2)}$ и $\left|V_{F}\right|$, что вызывает переход системы в нерезонансный режим (сплошная кривая на рис. 5). В этом случае значения $S \approx 1$ наблюдаются почти на всем интервале изменения угла $\varphi_{2}$. (Напомним, что все результаты, приведенные в работе, получены в предположении, что штарковские сдвиги компенсируются одним из упомянутых выше способов.) Вместе с тем на графиках $S\left(\varphi_{2}\right)$ присутствуют характерные особенности, а именно два резких минимума, где $S \approx 0$. Они сужаются с увеличением расстояния между КТ 1 и КТ 2, что говорит об их внутриструктурном происхождении. Увеличение расстояния $L_{y}$ уменьшает влияние заряженной КТ 3 на электрон-фотонный спектр структуры, приводя к ожидаемому снижению контраста. Сама функция $S\left(\varphi_{2}\right)$ демонстрирует осцилляции с амплитудой, уменьшающейся с ростом $L_{y}$. Но, как следует из рис. 5 , для $a=6$ существует угол поворота КТ $2 \varphi_{2} \approx 2 \mathrm{rad}$, при котором контраст сохраняет максимальное значение $S=1$ для всех трех расстояний $L_{y}$. Поскольку для более протяженной структуры с $a=8$ аналогичная точка отсутствует, то с учетом компенсации штарковских сдвигов данная особенность однозначно ассоциируется с эффектом Ферстера. Для состояния ТС 2 наблюдается похожая ситуация. Что же касается состояния двух КТ с энергией $\omega_{Q D}-V_{F}$ и соответствующего дублета Тависа-Каммингса, то здесь участки с высоким значением контраста (окрестности точек $\varphi_{2}=0$ и $\varphi_{2}=\pi$ ) характеризуются низкими величинами фототока, и поэтому его использование в качестве сенсорного состояния будет малоэффективным. В завершении нашего исследования укажем на то, что в дисперсионном режиме (без компенсации штарковских сдвигов) контраст убывает гораздо быстрее с расстоянием от структуры до КТ 3 [15].

Результаты, полученные нами, могут быть полезны при разработке оптических сенсорных устройств на основе одномерных фотонных кристаллов [20], использующих принцип субфотонной лазерной накачки в режиме сильного взаимодействия между КТ и фотоном [21].

\section{Заключение}

В настоящей работе рассмотрена модель квантовой наноструктуры на основе микрорезонатора с двумя заряженными КТ с оптическим субфотонным управлением, которая предназначена для детектирования отдельных электронов. Исследованы вопросы, связанные с влиянием кулоновских эффектов на точность измерения. Получено приближенное аналитическое выражение для среднего числа фотонов в моде микрорезонатора как функции частоты управляющего лазера и параметров системы. Рассчитаны зависимости контраста от энергии Ферстера, которая варьируется благодаря вращению одной из КТ, при полной компенсации внутриструктурных штарковских сдвигов. Показано, что при небольшом расстоянии между сенсорными КТ существуют конфигурации, для которых высокое значение контраста сохраняется даже на значительном удалении от структуры измеряемой КТ. Увеличение размера структуры приводит к подавлению взаимодействия Ферстера между сенсорными КТ, при этом указанные конфигурации отсутствуют. В целом, данные расчетов указывают на необходимость корректного учета как диагональных, так и недиагональных кулоновских матричных элементов гамильтониана при моделировании спектрального отклика таких систем. 


\section{Благодарности}

Автор выражает глубокую признательность И.Ю. Катееву в обсуждении результатов и подготовке рукописи.

\section{Финансирование работы}

Работа выполнена в рамках Государственного задания ФТИАН им. К.А. Валиева РАН Минобрнауки РФ по теме № FFNN-2022-0016 „Фундаментальные и прикладные исследования в области разработки методов высокоточного моделирования и контроля элементной базы квантовых компьютеров“.

\section{Конфликт интересов}

Автор заявляет, что у него нет конфликта интересов.

\section{Список литературы}

[1] B.A. Joyce, P.C. Kelires, A.G. Naumovets, D.D. Vvedensky. Quantum Dots: Fundamentals, Applications, and Frontiers. NATO Science Series (Springer, Dordrecht, 2003).

[2] S. Kiravittaya, M. Benyoucef, R. Zapf-Gottwick, A. Rastelli, O.G. Schmidt. Appl. Phys. Lett., 89, 233102 (2006). DOI: $10.1063 / 1.2399354$

[3] T. Mano, R. Nötzel, D. Zhou, G.J. Hamhuis, T.J. Eijkemans, J.H. Wolter. J. Appl. Phys., 97, 014304 (2005). DOI: $10.1063 / 1.1823578$

[4] T. van Lippen, R. Nötzel, G.J. Hamhuis, J.H. Wolter. J. Appl. Phys., 97, 044301 (2005). DOI: 10.1063/1.1840098

[5] A. Walmsley. Science, 348, 525 (2015). DOI: $10.1126 /$ science.aab0097

[6] L. Zhang, C.-H. Teng, T.A. Hill, L.-K. Lee, P.-C. Ku, H. Deng. Appl. Phys. Lett., 103, 192114 (2013).

DOI: $10.1063 / 1.4830000$

[7] A. Dousse, J. Suffczynski, O. Krebs, A. Beveratos, A. Lemaitre, I. Sagnes, J. Bloch, P. Voisin, P. Senellart. Appl. Phys. Lett., 97, 081104 (2010). DOI: 10.1063/1.3475487

[8] K.K. Yadavalli, A.O. Orlov, J.P. Timler, C.S. Lent, G.L. Snider. Nanotechnology, 18, 375401 (2007).

[9] M.P. Bakker, A.V. Barve, T. Ruytenberg, W. Löffler, L.A. Coldren, D. Bouwmeester, van M.P. Exter. Phys. Rev. B, 91, 115319 (2015). DOI: 10.1103/PhysRevB.91.115319

[10] А.В. Цуканов. Опт. и спектр., 123, 591 (2017). DOI: $10.7868 / \mathrm{S} 0030403417100245$ [A.V. Tsukanov. Opt. Spectrosc., 123, 591 (2017). DOI: $10.1134 / \mathrm{S} 0030400 \mathrm{X} 17100241]$

[11] C. Barthel, M. Kjærgaard, J. Medford, M. Stopa, C.M. Marcus, M.P. Hanson, A.C. Gossard. Phys. Rev. B, 81, 161308(R) (2010). DOI: 10.1103/PhysRevB.81.161308

[12] А.В. Цуканов, И.Ю. Катеев. Микроэлектроника, 49, 83 (2020). DOI: 10.31857/S0544126920020088 [A.V. Tsukanov, I.Yu. Kateev. Russian Microelectronics, 49, 77 (2020). DOI: $10.1134 / \mathrm{S} 1063739720020080]$.

[13] C.B. Simmons, M. Thalakulam, N. Shaji, L.J. Klein, H. Qin, R.H. Blick, D.E. Savage, M.G. Lagally, S.N. Coppersmith, M.A. Eriksson. Appl. Phys. Lett., 91, 213103 (2007). DOI: $10.1063 / 1.2816331$
[14] А.В. Цуканов, И.Ю. Катеев. Микроэлектроника, 50, 83 (2021). DOI: 10.31857/S0544126921020095 [A.V. Tsukanov, I.Yu. Kateev. Russian Microelectronics, 50, 75 (2021). DOI: $10.1134 / \mathrm{S} 1063739721020098]$.

[15] А.В. Цуканов. Квантовая электроника, 51, 84 (2021). [A.V. Tsukanov. Quant. Electron., 51, 84 (2021). DOI: 10.1070/QEL17441].

[16] R. Ohta, Y. Ota, M. Nomura, N. Kumagai, S. Ishida, S. Iwamoto, Y. Arakawa. Appl. Phys. Lett., 98, 173104 (2011). DOI: $10.1063 / 1.3579535$

[17] D. Yang, B. Duan, X. Liu, A. Wang, X. Li, Y. Ji. Micromachines, 11, 72 (2020). DOI: 10.3390/mi11010072

[18] П.А. Головинский. ФТП, 48, 781 (2014). [P.A. Golovinskii. Semiconductors, 48, 760 (2014). DOI: $10.1134 / \mathrm{S} 1063782614060104]$.

[19] M. Ciurla, J. Adamowski, B. Szafran, S. Bednarek. Physica E, 15, 261 (2002). DOI: 10.1016/S1386-9477(02)00572-6

[20] D.-Q. Yang, X. Liu, X.-G. Li, B. Duan, A.-Q. Wang, Y.F. Xiao. Journal of Semiconductors, 42, 023103 (2021). DOI: $10.1088 / 1674-4926 / 42 / 2 / 023103$

[21] D.A. Rasero, A.A. Portacio, P.E. Villamil, B.A. Rodríguez. Physica E, 129, 114645 (2021). DOI: $10.1016 /$ j.physe.2021.114645 